\title{
¿Quién se beneficia de las certificaciones de café orgánico? \\ El caso de los campesinos de La Sepultura, Chiapas
}

\section{Who Benefits from Organic Coffee Certifications? \\ The Case of Peasants from La Sepultura, Chiapas}

\author{
Iván Tlaloc Soleto Polanco \\ Investigador independiente \\ email: ivan_0679@hotmail.com \\ ORCID: orcid.org/0000-0001-5916-6660 \\ Juana Cruz-Morales \\ Universidad Autónoma Chapingo \\ cruz_juanam@yahoo.com.mx \\ ORCID: orcid.org/0000-0002-5410-2702
}

Resumen:

El objetivo de esta investigación es valorar si la participación en programas de certificación orgánica es una herramienta exitosa para sacar de la pobreza a los pequeños productores del sur mediante la mejora de sus medios de vida y la reducción de su vulnerabilidad. Los métodos de investigación consisten en una amplia revisión de la literatura pertinente y en un estudio de caso. Los hallazgos muestran que los productores siguen siendo el eslabón más débil de la cadena de valor, al igual que ocurre con las cadenas comerciales de café convencional y, por lo tanto, no están obteniendo los beneficios esperados.

Palabras clave: medios de vida sostenible, vulnerabilidad, cadenas de valor, roya, ONG.

\section{Abstract:}

This research aims to assess whether participation in organic certification schemes is a successful tool to bring small-scale producers from the South out of poverty through improving their livelihood and reducing their vulnerability. The research methodology consists of an extensive revision of the related literature, as well as a case study. Findings demonstrate that producers continue being the weakest link in the value chain, as happens in the commercial chains of conventional coffee, and are therefore not obtaining the expected benefits.

Keywords: sustainable livelihood, vulnerability, value chains, coffee leaf rust, NGO. 


\section{Introducción}

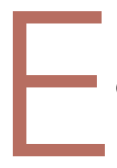

I café es el producto tropical más importante; representa al menos la mitad de las exportaciones netas en ese ramo y es el segundo producto más valioso exportado por los países del sur, solo por detrás del petróleo (Hallam 2003). Su producción involucra de forma directa aproximadamente a 23 millones de familias en países australes, ${ }^{1}$ e indirectamente alrededor de 100 millones participan en actividades relacionadas con su presencia en el mercado (Hallam 2003).

Este cultivo se introdujo en las colonias alrededor del siglo XVIII mediante grandes plantaciones controladas por terratenientes, para su posterior venta en los comercios internacionales. Ha sido considerado, junto con el azúcar, uno de los principales cultivos del colonialismo europeo. En muchas colonias, debido al aumento de demanda, se importaron esclavos desde África para trabajar en las plantaciones de esta rubiácea. En definitiva, su cultivo ha estado históricamente presente en los países tropicales del sur para su consumo en los países del norte, generalmente basado en la explotación de los trabajadores y la tierra (Wild 2005).

En la actualidad, una gran proporción de los pequeños cafeticultores (PC) que viven en los países sureños tienen que enfrentarse normalmente a múltiples desafíos para contar con medios de vida dignos, tales como precios bajos, escaso o nulo poder de negociación, obstáculos para entrar en los mercados internacionales o la falta de acceso a instituciones de crédito oficiales (Ponte 2002). A lo anterior se suman sus desventajas para competir en términos de productividad frente a las grandes plantaciones y empresas trasnacionales. Además, debido a su limitada capacidad financiera y a la naturaleza volátil del mercado de esta semilla, los PC están expuestos a riesgos tales como la fluctuación de precios y los desastres naturales (CEPAL 2002).

En muchos casos, esta situación ha empujado a los PC a llevar a cabo prácticas agrícolas nocivas para el medioambiente para poder obtener mayores beneficios. También debido a un bajo conocimiento técnico en el uso de fertilizantes y pesticidas que a largo plazo agrava el problema (Schroth et al. 2009). Aunque es importante recalcar que en muchas regiones cafetaleras los PC no pueden permitirse la compra de insumos agroquímicos.

\footnotetext{
1 Brasil, Vietnam, Colombia, Indonesia, Etiopía, India, Honduras, Uganda, México, Guatemala, Perú, Nicaragua, Costa de Marfil, Costa Rica, Kenia, El Salvador y Ecuador.
} 
Algunos de los cafeticultores viven en áreas naturales de protección especial. Un ejemplo de este tipo de áreas son las reservas de la biosfera:

áreas que buscan impulsar armónicamente la integración de las poblaciones y la naturaleza, a fin de promover un desarrollo sostenible mediante un diálogo participativo, el intercambio de conocimiento, la reducción de la pobreza, la mejora del bienestar, el respeto a los valores culturales y la capacidad de adaptación de la sociedad ante los cambios (UNESCO 2015).

Esta situación conlleva desafíos extra a los productores debido a las restricciones legales impuestas en algunas prácticas agrícolas.

Las comunidades en donde se efectuó el estudio corresponden a este contexto debido a que se localizan en la reserva de la biosfera La Sepultura (REBISE). Su pertenencia a la zona de amortiguamiento de la REBISE supone que sus habitantes se sometan a prohibiciones en sus prácticas agropecuarias y forestales como la quema y el desbroce, la deforestación y el uso de agroquímicos. En este caso, tales restricciones son supervisadas estrictamente debido a que las comunidades se ubican en el borde de la zona núcleo de la reserva (Brunel y García-Barrios 2011).

Sin embargo, la situación de pobreza en que se hallan la mayor parte de los productores en la REBISE propicia —al igual que en la mayoría de poblados de Chiapas, donde $74.5 \%$ de la población está por debajo del umbral de pobreza (CONEVAL 2012) - , prácticas de sobrexplotación y otras desfavorables en el manejo de sus propios recursos naturales, de los cuales son extremadamente dependientes. Para los productores en las comunidades estudiadas, el café representa un papel esencial en sus medios de vida; la mayor parte de sus ingresos provienen de su venta. Además, debido a las condiciones de altitud y pendiente de sus parcelas, probablemente sea la mejor opción productiva (Bartra et al. 2003).

En este contexto, diferentes instituciones presentaron en la REBISE, al igual que en otras áreas protegidas de Centroamérica, un modelo integrado de negocio basado en la certificación de café orgánico, «C.A.F.E Practices», como una herramienta de desarrollo convergente entre la conservación de la reserva y la mejora de los medios de vida de sus habitantes. Esta estrategia impulsada por organizaciones no gubernamentales (ONG) internacionales, empresas privadas y las autoridades de la reserva tenía como objetivo la producción orgánica de café, lo cual les ayudaría a enfrentar sus principales problemas en el corto y medio plazo por medio de la elevación de precios en los mercados de café orgánico y 
el desarrollo de relaciones comerciales más estables y justas y, en consecuencia, reducir su pobreza. Por otro lado, se asume que los métodos de cultivo orgánico aseguran la conservación de la biodiversidad, los suelos y la calidad del agua para las siguientes generaciones (Barrett et al. 2002). Por lo tanto, este modelo fue adoptado institucionalmente por la Comisión Nacional de Áreas Naturales Protegidas (CONANP) para lograr los objetivos de conservación de la reserva al frenar la degradación de los recursos naturales provocada por las malas prácticas agrícolas.

Sin embargo, en los últimos años se han alzado voces críticas contra este tipo de estándares como estrategia comercial. Estas voces discordantes consideran que tales estándares tienden a desarrollarse en apego a los intereses de los consumidores del primer mundo y el resto de actores del final de la cadena de valor; además, que son impuestos por actores externos e intermediarios, con poca o ninguna participación de los productores locales. Por lo tanto, estos últimos podrían quedar sin los beneficios esperados, y no cumplirse el intento de sacar de la pobreza a los pequeños productores (González y Nigh 2005).

Algunos estudios de evaluación de impacto sugieren que no debería darse por hecho la efectividad de la participación en esquemas de certificación como una estrategia para mejorar los modos de vida de los pequeños productores, porque no siempre hay una relación de causalidad entre esa participación y la mejora de los modos de vida de los involucrados (Beuchelt y Zeller 2011).

Este debate demuestra que hay una falta de información en los efectos reales de la participación en certificaciones orgánicas de café; por lo tanto, es importante ponderar los cambios experimentados entre quienes operan bajo dichos esquemas para poder ampliar el conocimiento sobre sus consecuencias en lo tocante a los medios de vida de los pequeños productores en áreas protegidas. De este modo, los pequeños cafeticultores y otros actores involucrados en la producción de café podrían usar este conocimiento para definir su participación en esas certificaciones de una manera exitosa.

Basado en las percepciones de los actores implicados, este estudio intenta valorar los cambios, no solo en bienes tangibles como los precios, sino también otros, tales como la forma en que los productores hacen frente a los riesgos y vulnerabilidades o si ahora tienen relaciones comerciales más estables, comparado con su propia situación antes de participar en la cadena de valor de café orgánico certificado. 
Dado que no hay estudios específicos sobre el tema en la región, parece necesario analizar los cambios experimentados por los pequeños productores de café, para así poder determinar si dicha participación representa una estrategia válida para lo que se busca, y por qué, y de ese modo ampliar la perspectiva con base en las pruebas de este caso, de las condiciones y los factores de éxito de dicha estrategia.

\section{Materiales y métodos}

El método de investigación utilizado fue un estudio de caso (Cohen y Manion 1995). Esta herramienta permitió analizar intensivamente el vínculo entre los cambios en los medios de vida de los productores y su participación en la certificación orgánica de café en la REBISE.

El estudio se llevó a cabo durante cuatro meses, en 2015. Se diseñó un marco analítico similar al utilizado al propósito por diversos autores (Bacon 2005, 2015, Beuchelt y Zeller 2011, Fitter y Kaplinsky 2001, Kilian et al. 2006). Con este fin se elaboraron un conjunto de indicadores mediante una combinación del enfoque medios de vida sostenibles o sustainable livelihood approach (SLA), desarrollado por el Departamento para el Desarrollo Internacional (DFID) (Carney 1999) y un análisis de la cadena de valor (Kaplinski y Morris 2000).

El análisis de la cadena de valor pone énfasis en las estructuras y los procesos que influyen en los medios de vida de los productores, particularmente en el entorno del mercado orgánico. Este enfoque es usado para determinar si la participación en las cadenas de valor orgánicas lleva a los productores certificados a obtener precios más estables y altos, y si su poder de negociación ha aumentado. Mientras que los medios de subsistencia, sus estrategias, resultados, y el contexto de vulnerabilidad es estudiado por medio del SLA, el cual permite una mejor comprensión de los medios de vida.

Como expone el SLA, los medios de vida y el contexto de vulnerabilidad están estrechamente interrelacionados debido a que la acumulación de bienes reduce los impactos negativos de los choques externos que sufren los productores, y al mismo tiempo estos choques tienen la capacidad de destruir los bienes de los productores. Por lo tanto, se desarrollaron indicadores indirectos para poder valorar los cambios en los distintos tipos de capital (natural, social, financiero, físico y humano) de los productores y si efectivamente dichos cambios han fortalecido 
las estrategias para hacer frente a los posibles riesgos que afectan a los productores de café. Además, las causas de estos cambios son analizadas para poder dilucidar si estos son atribuibles a la participación en las certificaciones orgánicas.

Cuando analizamos los medios de vida tenemos que adaptar los indicadores al contexto local. Los indicadores sugeridos por la literatura fueron complementados con otros extraídos de un manual sobre medios de vida que diseñaron investigadores locales para abordar específicamente el área de estudio de referencia (Parra 2011). Algunos de los utilizados fueron: precio por quintal de café, capacidad de ahorro al final de la temporada, obtención de subsidio, fertilidad del suelo, conocimientos sobre agricultura orgánica y mercados internacionales de café, infraestructura para el procesamiento primario del café, relación con el gobierno y otras organizaciones nacionales e internacionales, entre otros. Estos indicadores se enumeran más adelante, en la tabla 2 del apartado de resultados.

El principal instrumento para recopilar la información fue la entrevista semiestructurada. Consiste en tener una guía de entrevista con preguntas agrupadas por temas o categorías, con base en los objetivos de estudio (Díaz-Bravo et al. 2013). También se tomaron en cuenta las conversaciones informales y la observación directa con el fin de asegurar métodos comparativos. Se compiló la información por medio de entrevistas con los principales actores involucrados en la producción de café en el área de estudio.

Tres Picos y Nueva Independencia, las dos comunidades donde se realizó el trabajo, han participado en este modelo de comercialización desde hace más de 10 años. Ambas tienen un número relativamente pequeño de productores, lo que facilitó la entrevista a una gran proporción de los productores. Se hizo una tipología de productores entrevistados en relación con el tamaño de sus tierras (tabla 1), y fueron seleccionados aleatoriamente dentro de esas categorías. En Tres Picos hay 51 productores, de los cuales 26 fueron entrevistados (51\%). En Nueva Independencia se consultó a 12 de sus 31 productores (39\%). Para triangular la información y tener una visión más amplia de la situación, se hizo una revisión de datos secundarios basados en estudios anteriores a las certificaciones (informes técnicos), y se aplicó el cuestionario a los principales actores involucrados en la producción de café; a investigadores de las universidades locales (CIMSUR y Universidad Autónoma Chapingo), miembros de ONG (Conservation International y The Nature Conservancy) y al director de la REBISE. 
Tabla 1. Clasificación de los productores respecto del tamaño de sus cafetales.

\begin{tabular}{|c|c|}
\hline Tamaño Pequeño & $<$ de 2 hectáreas de café \\
\hline Tamaño Medio & De 2 a 5 hectáreas de café \\
\hline Tamaño grande & $>$ de 5 hectáreas de café \\
\hline
\end{tabular}

Fuente: Elaboración propia.

La lógica de investigación (figura 1) consistió en ligar las hipótesis o preguntas del estudio con las de las entrevistas para poder facilitar el análisis de las transcripciones. En consecuencia, las hipótesis son divididas en categorías, que a su vez son separadas en especificaciones o indicadores. Finalmente, las preguntas de las entrevistas responden a cada uno de estos indicadores. Más que un proceso lineal, es un ciclo donde los indicadores, después del análisis, ayudan a responder a las hipótesis, lo que finalmente abona a cumplir el objetivo del estudio.

Figura 1: Lógica de la investigación.

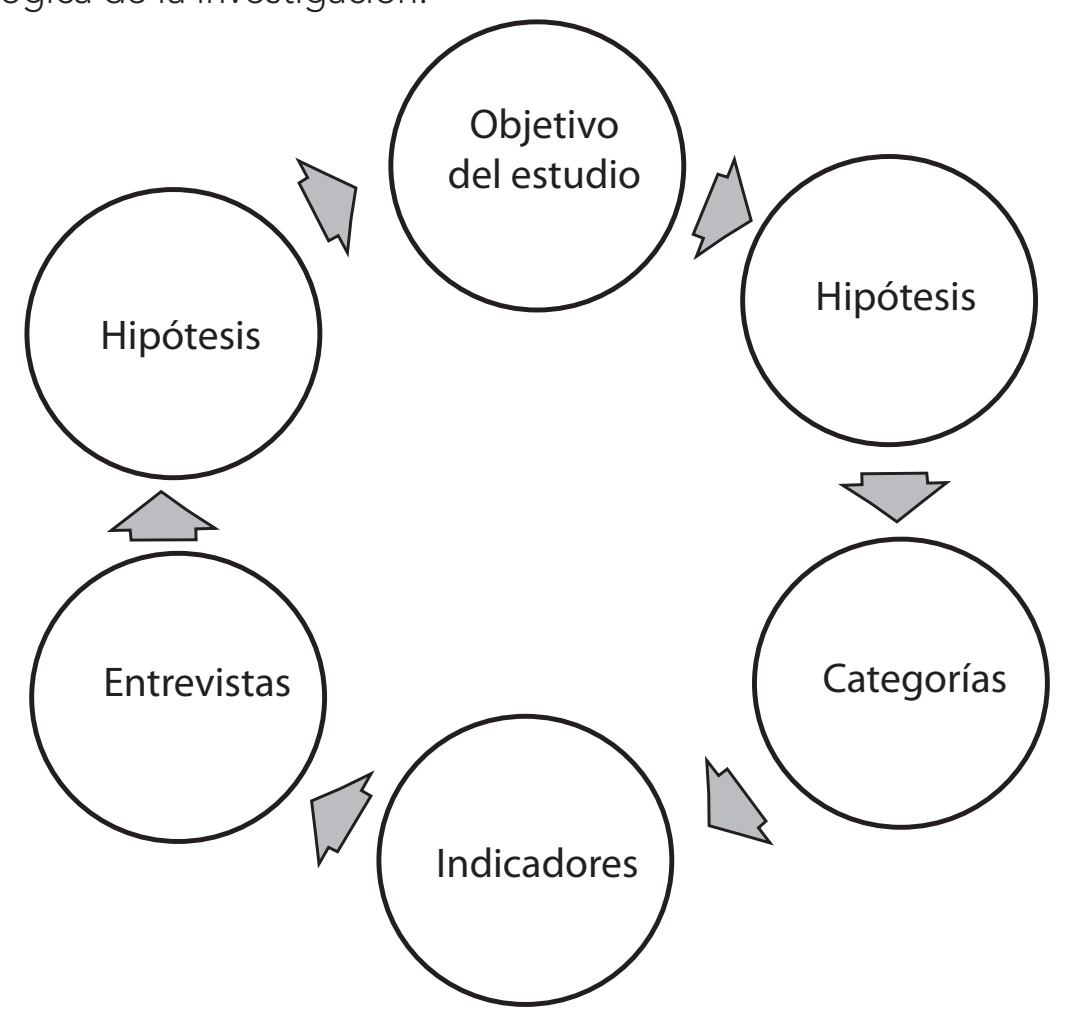

Fuente: Elaboración propia. 


\section{Resultados}

Los dos ejidos ${ }^{2}$ forman parte del área de influencia del municipio de Villaflores, el cual a su vez está subordinado a la capital del estado, Tuxtla Gutiérrez (Camacho et al. 2002).

Los habitantes de la reserva han estado cultivando café desde antes de que esta fuera establecida, en 1995. Al principio, la producción de café era dedicada únicamente al consumo, a causa de la falta de mercado para este tipo de cultivos. En esta parte de la REBISE, las condiciones ambientales son favorables (clima, altitud y vegetación) para el cultivo de café de la variedad arábica, el cual tradicionalmente se cultiva a la sombra de un dosel arbóreo, principalmente del bosque nuboso. En las plantaciones cafetaleras hay una gran variedad de árboles tropicales, tales como chalum (Inga vera) o vaqueta (Ulmus mexicana), los cuales son hogar de diversas especies de aves y otro tipo de fauna silvestre (Camacho et al. 2002).

Actualmente, la gran mayoría de los habitantes de Tres Picos y Nueva Independencia cultivan café en sus tierras. Por un lado, debido a las restricciones impuestas por las autoridades de la reserva sobre otro tipo de actividades productivas, como el aprovechamiento forestal o la ganadería. Por otra parte, gracias a los buenos precios que lograron fijarse ${ }^{3}$ (entrevista a un productor de café, 6 de abril del 2015). Además, es considerado por las autoridades ambientales mexicanas como sostenible y con pocos efectos sobre el ambiente (comunicación personal de la Dirección de la REBISE, 2016).

Las características de los productores son muy homogéneas. En general, los cafeticultores son hombres de entre 40 y 60 años. La gran mayoría de ellos saben leer y escribir, aunque su nivel de estudios es básico.

En nuestros días, el cultivo de dicha rubiácea es una de las principales actividades productivas. Para un porcentaje muy alto de los habitantes de la región representa el grueso de sus ingresos; entre los productores certificados orgánicamente llega a ser alrededor de $60 \%$ a $70 \%$ de sus ingresos, y los complementan con producciones de maíz, palma camedor o resina de pino.

2 El ejido es una comunidad campesina creada por la distribución de tierras bajo la reforma agraria (1917-1992). Los ejidatarios recibieron tierras para su uso y usufructo, pero sin dejar de ser propiedad de la nación. Los derechos son heredables, sujetos a ciertas reglas, pero la tierra no se puede vender. Bajo esta forma jurídica de tenencia de la tierra se buscó propiciar una gestión compartida de los recursos comunes (Randall 1996).

${ }^{3}$ En el 2011 llegaron a recibir hasta 90 pesos por kg de café. 
Las plantaciones son trabajadas principalmente por los miembros de la familia. Los hombres se hacen cargo del trabajo agrícola. Las mujeres también están involucradas en el proceso final, en el despulpado, lavado y secado de la semilla. Obtienen en promedio $550 \mathrm{~kg}$ por hectárea en un buen año (entrevistas a productores, 6 de abril de 2015).

\section{Desarrollo de la certificación orgánica en la REBISE}

Conservation International ${ }^{4}(\mathrm{Cl})$, la Agencia Estadounidense de Cooperación y Desarrollo (USAID) y la Comisión Nacional de Áreas Naturales Protegidas (CONANP) establecieron un modelo de negocio basado en las certificaciones orgánicas para integrar a los productores en una cadena orgánica de valor internacional y promover la producción de café de sombra en la zona (véanse figuras 2 y 3 ). Este modelo de negocio buscaba fomentar en los productores la adopción de buenas prácticas ambientales en sus tierras. A cambio, podrían exportar su café al mercado internacional a precios más altos que los del producto convencional. Para realizar la transición de café de sombra convencional a café de sombra orgánico se impartieron, en colaboración con El Colegio de la Frontera Sur (Ecosur), cursos de capacitación (escuelas de campo) en prácticas orgánicas junto con asistencia técnica para formar una cooperativa, Unión de Productores de la Sierra de Villaflores (UPROSIVI), integrada en el 2001, y que cuenta con 151 miembros.

Para poder tener una demanda constante se estableció una alianza público-privada. $\mathrm{Cl}$ se asoció con Starbucks como comprador y como donante en el programa, y de ese modo se lograba complementar los fondos recibidos de la USAID. Starbucks añadió sus propios estándares para poder comercializar el café bajo su propia marca (Coffee and Farmer Equity practices ${ }^{5}$ ). Dichos estándares buscaban tanto la conservación ambiental como la mejora en los medios de vida de los productores. La certificación, realizada por la certificadora mexicana OCIA-CERTIMEX, permitiría a los productores exportar su café a Europa, Estados Unidos y Japón.

En principio, el café orgánico era vendido directamente desde la cooperativa a Starbucks, con la intermediación de $\mathrm{Cl}$ entre ambos debido a la falta de capacidades y conocimientos de los productores para exportarlo ellos mismos. Sin embar-

4 Conservation International es una ONG ambientalista con sede en Estados Unidos cuyo principal objetivo es la protección de la biodiversidad del planeta.

5 <https://www.scsglobalservices.com/starbucks-cafe-practices> 
Figura 2: Representación gráfica de cómo operaban inicialmente las certificaciones orgánicas. Las flechas bidireccionales indican una relación de doble vía y las unidireccionales una relación directa.

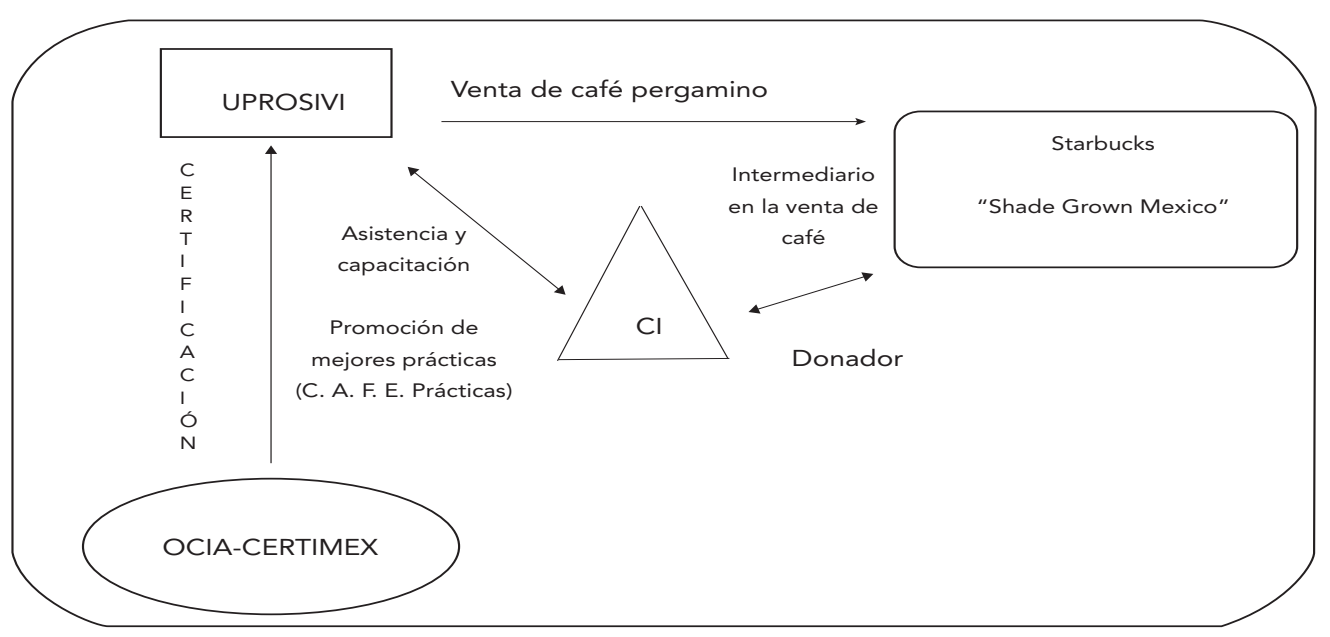

Fuente: Elaboración propia con base en trabajo de campo.

Figura 3: Línea de tiempo del proceso de certificación de orgánica.

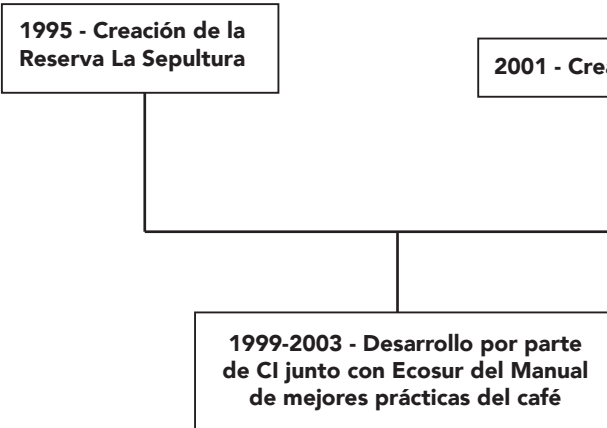

2005 - Starbucks lanza C. A. F. E

practices. Inicio de compra a

AMSA por parte de Starbucks

Fuente: Elaboración propia con base en trabajo de campo.

go, hubo un desacuerdo entre la compañía y los productores sobre cómo exportar el café. Este desacuerdo se basaba en problemas en la regularidad, calidad y entrega del grano. Starbucks indicó su necesidad de reducir los costos de transacción, y para ello se introdujo a otra empresa en el modelo: Agro Industrias Unidas de México ${ }^{6}$ (AMSA), como intermediaria entre los productores y Starbucks. Por lo tanto, el modelo inicial desapareció y se dio paso a otro escenario (figura 4) en que

${ }^{6}$ AMSA, conocido también como Omnicafé, es parte del grupo ECOM-Trading (con sede en Suiza) de la familia Esteve y dedicado a las commodities. Le pertenece también el importador Atlantic Coffee (Renard 2008). 
Figura 4: Representación gráfica de cómo operan las certificaciones orgánicas.

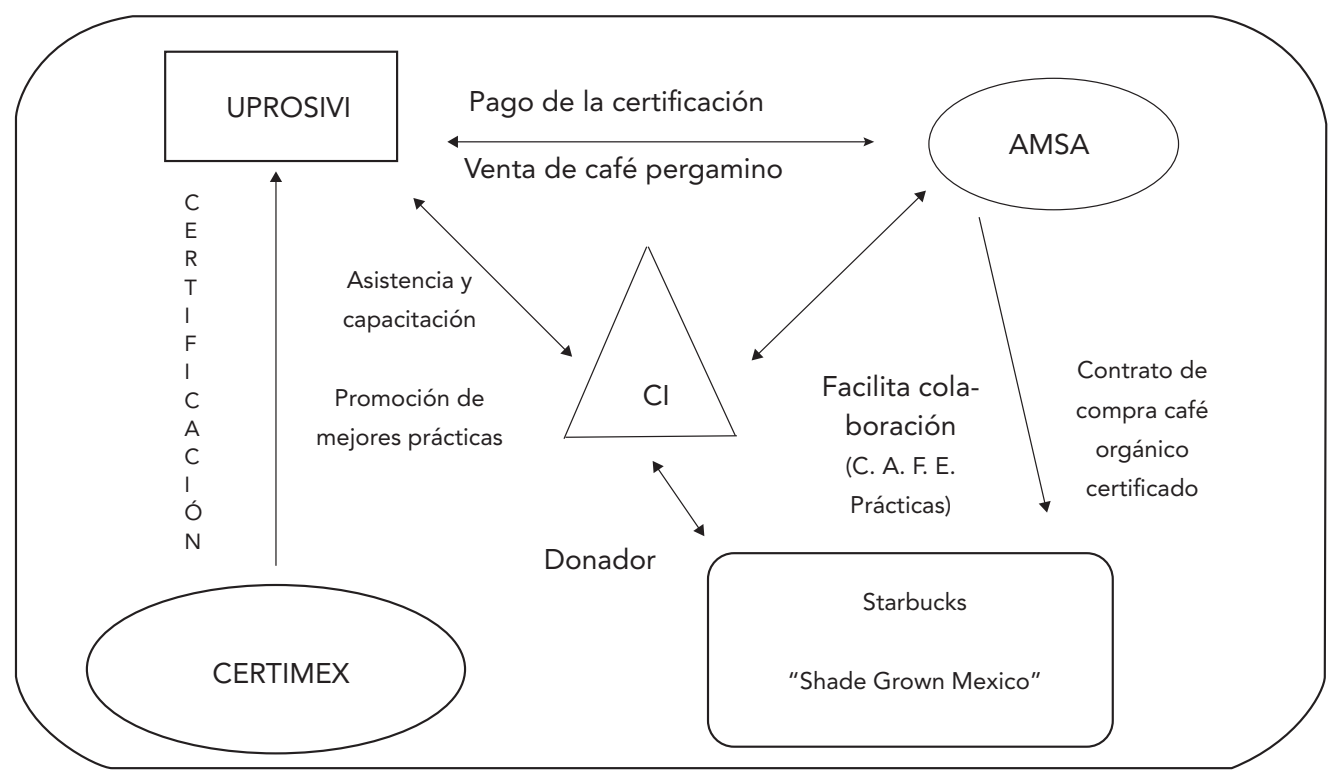

Fuente: Elaboración propia en base al trabajo de campo.

los productores de café certificados vendían individualmente su café pergamino a la compañía exportadora, que es básicamente el único comprador disponible en la zona. AMSA también cubre los costos de la certificación, y los descuenta del precio de compra; de ese modo se obtienen los documentos que acreditan la certificación, ya que los productores no pueden solventar el costo anual de la certificación en un solo pago. Además, la falta de acceso a créditos en instituciones oficiales ha llevado a que los productores busquen en AMSA también un agente financiador de avío; AMSA otorga créditos para invertir en la producción a un interés considerablemente alto, de $10 \%$ a $15 \%$ anual, de acuerdo con lo mencionado por los productores. Este programa se ejerció durante el periodo 2004-2014.

\section{Impactos en los medios de vida y vulnerabilidad}

La tabla 2 muestra los principales hallazgos respecto de cómo impacta en los medios de vida la participación en certificaciones orgánicas. Los resultados son divididos en los distintos tipos de capitales, los cuales están basados en los indicadores desarrollados en el marco analítico. Como se mencionó previamente, los productores fueron divididos en tres grupos según el tamaño de sus plantacio- 
Tabla 2. Impactos de la participación en certificaciones orgánicas en los distintos capitales.

\begin{tabular}{|c|c|c|c|c|c|}
\hline & Capital natural & Capital financiero & Capital humano & Capital social & Capital físico \\
\hline $\begin{array}{l}\text { Impactos } \\
\text { positivos }\end{array}$ & $\begin{array}{l}\text { — Fertilidad del } \\
\text { suelo } \\
\text { — Calidad del } \\
\text { agua } \\
\text { — Biodiversidad }\end{array}$ & $\begin{array}{l}\text { - Ayudas por parte } \\
\text { del gobierno }\end{array}$ & $\begin{array}{l}\text { - Conocimiento } \\
\text { adquirido sobre } \\
\text { manejo orgánico }\end{array}$ & $\begin{array}{l}\text { — Relación con las } \\
\text { instituciones (CONANF, } \\
\text { ONG, etc.) }\end{array}$ & $\begin{array}{l}\text { - Herramientas e } \\
\text { infraestructuras } \\
\text { (despulpadora y } \\
\text { patio de secado) }\end{array}$ \\
\hline Sin impactos & & $\begin{array}{l}\text { - Precios } \\
\text { - Acceso al crédito }\end{array}$ & $\begin{array}{l}\text { - Mercados } \\
\text { internacionales de } \\
\text { café } \\
\text { — Proceso de } \\
\text { certificación } \\
\text { — Habilidades } \\
\text { de negocio y } \\
\text { organización }\end{array}$ & $\begin{array}{l}\text { - Relación con los } \\
\text { compradores }\end{array}$ & \\
\hline
\end{tabular}

Fuente: Elaboración propia.

nes. Sin embargo, los resultados no confirman ninguna relación causal entre ese factor y los impactos experimentados.

Entre las principales causas de vulnerabilidad de los productores se encuentran las plagas y enfermedades (roya ${ }^{7}$ ), las catástrofes naturales (huracán en el 2011), la inestabilidad financiera que causan las fluctuaciones internacionales del precio del café Arábica y la pobre gobernanza de la cadena de valor.

Al día de hoy, la roya parece ser el principal problema al que se enfrentan los productores. A pesar de que ha sido detectada en la zona desde hace más de 10 años, desde hace tres se ha vuelto más virulenta y se ha esparcido a todas las plantaciones, causando grandes pérdidas en los cafetales. En 2015 las pérdidas por esa causa fueron estimadas en $60 \%$ respecto de la producción del año anterior. Teniendo en cuenta que los productores dependen básicamente de la producción de café, ese porcentaje de pérdidas es una amenaza que podría llevar al colapso de la economía familiar. A lo anterior hay que sumarle el problema de los precios bajos y fluctuantes del grano. La gran mayoría de los productores

\footnotetext{
7 Enfermedad del café causada por el hongo Hemileia vastatrix. Los síntomas son pequeñas manchas de color amarillo pálido en las superficies superiores de las hojas, que causan defoliación prematura, y por lo tanto reduce la capacidad fotosintética y debilita el árbol. La roya del café disminuye drásticamente el rendimiento, y se considera como la enfermedad de café económicamente más importante en el mundo (Thurston 1998).
} 
se quejan de los precios extremadamente bajos (para 2015 fluctuó entre $39^{8}$ y 45 pesos por kilo), ligeramente más altos que los recibidos por los cafetaleros de café convencional. Aunque los productores son conscientes de que el café orgánico es más caro, explican que la razón para obtener precios tan bajos es su inexistente poder de negociación.

Como ya ha sido descrito, una de las principales causas de vulnerabilidad son los precios. AMSA, el intermediario entre los cafeticultores y Starbucks, ejerce su asimétrico poder de negociación para comprar a precios reducidos. Esta asimetría es el resultado de diversos factores: en primer lugar, es el único comprador en la zona que puede absorber toda la oferta. Además, debido a que los cafeticultores tienen como principal fuente de ingresos la venta del grano, se ven obligados a desplazar su producto tan pronto como lo cosechan para poder comprar comida, pagar las facturas, etcétera. Debido entonces a sus limitadas opciones y a su nulo poder de negociación, se ven obligados a aceptar el precio que les ofrezcan. Todos los entrevistados encuentran estos precios injustos y argumentan que la compañía está jugando el papel de «coyote ${ }^{9}$ verde», lo que significa que compra el café al mismo precio que el convencional, gracias a su poder de negociación, para después venderlo como orgánico a Starbucks u otras empresas. En este punto es conveniente mencionar que el autor trató de conocer el punto de vista de la empresa, pero AMSA rechazó ser entrevistada en varias ocasiones.

En definitiva, podemos concluir que los resultados de la investigación no confirman un impacto sustancial en los bienes o estrategias contra las principales causas de vulnerabilidad desde que los productores participan en certificaciones orgánicas de café.

\section{Asimetría en la cadena de valor de café orgánico}

Los motivos por los cuales la compañía exportadora, que monopoliza el mercado del café en la región (Mariscal 2010), paga precios más bajos de los esperados son los siguientes:

- Escaso poder de negociación por parte de los productores debido a la falta de una organización social que les permita hacer acopio de café y venderlo colectivamente, y obtener así mejores precios. Cuando se llevó a cabo la

\footnotetext{
8 En la fecha del estudio el precio equivaldría a 2.88 y 3.33 dólares por kilo.

9 Palabra coloquial mexicana para intermediarios, con una connotación negativa.
} 
transición de café convencional a orgánico no se hizo un proyecto a largo plazo que les permitiera desarrollar sus capacidades cooperativas, solo se estableció una cooperativa para que pudieran recibir ciertos apoyos puntuales por parte del gobierno, como un patio de secado.

- Las promesas de mejores precios por su café y la presión de las autoridades de la reserva para producir café de sombra les ha llevado a especializarse en café con una tendencia al monocultivo. Esta situación crea una necesidad de vender el café individualmente tan pronto como lo cosechan, debido la falta de fuentes alternativas de ingresos y a la mala producción causada por la roya.

- Incapacidad para encontrar otras opciones de compradores.

- Es la empresa exportadora la que "paga» los costos de certificación y obtiene los papeles que lo certifican como orgánico, y en consecuencia lo exporta como tal. Sin embargo, aunque los productores saben que son orgánicos, no tienen forma de demostrarlo porque el certificado año tras año queda bajo el poder de AMSA.

Las observaciones anteriores contradicen claramente la suposición sobre cómo los productores certificados orgánicamente mejoran su posición en la cadena de valor al obtener precios más altos y, por lo tanto, mejores rentas, y con ello escapar de la pobreza (Bacon 2005, Sustaineo 2013).

\section{Comparación de la situación en la REBISE con otros estudios}

Los resultados de la investigación no indican que al participar en este tipo de certificaciones orgánicas mejoren sustancialmente los medios de vida de los productores y se reduzca su vulnerabilidad.

Empezando por el complicado y costoso proceso burocrático de la certificación, que los pequeños productores de café tienen que superar para poder conseguir la certificación orgánica, y también la dificultad para alcanzar los mercados internacionales. Los resultados parecen estar en la misma línea que las publicaciones de otros investigadores (Allen y Kovach 2000, Bartra 2006, González y Nigh 2005), quienes declaran que el proceso de certificación es complicado y caro para los pequeños productores del sur. Esta situación los ha llevado a una gran dependencia de agentes externos para cumplir con los estándares orgáni- 
cos, y también para recibir apoyo económico a la hora de cubrir los costos de la certificación y el periodo de transición hacia la producción orgánica.

En lo relativo a las mejoras en el capital social y la contribución al desarrollo de capacidades gracias a la creación de cooperativas, los resultados de este estudio están más en concordancia con González y Nigh (2005) que con la opinión de Bacon (2005), quien afirma que la participación en certificaciones orgánicas de café tiene un impacto positivo en el capital social de los productores. González y Nigh argumentan que la formación de cooperativas mediante enfoques verticales no garantiza el trabajo colectivo de manera apropiada. Al contrario, las cooperativas de café orgánico más exitosas son aquellas que emplean de manera fuertemente democrática la toma de decisiones y tienen una organización política participativa.

La revisión de literatura también identifica como impacto positivo la conservación de los recursos naturales de los productores (Bacon 2005, Beuchelt y Zeller 2011, Kilian et al. 2006, Paschall 2013, Sustaineo 2013); dicha exposición es consistente con los resultados de este estudio. El propio gestor de la reserva aseguró durante la entrevista que las certificaciones orgánicas solo habían conseguido su objetivo desde el punto de vista de la conservación y que la calidad del agua, la fertilidad del suelo y la biodiversidad arbórea en las comunidades siguen en buen estado.

Además, la mejora en la educación de los cafeticultores parece estar verificada por los resultados obtenidos durante el estudio de caso. Esto puede ser explicado por los cursos de capacitación proporcionados a los productores sobre prácticas orgánicas. No obstante, según los resultados obtenidos todavía existe un déficit de conocimiento entre los productores certificados en lo relativo al proceso de certificación y los mercados internacionales de café, incluidos los orgánicos. La gran mayoría de los productores reconocen su incapacidad para entender y acceder a los mercados orgánicos de café. Por consiguiente, los resultados del estudio de caso niegan parte de los supuestos beneficios sociales y económicos (Bacon 2005, Paschall 2013, Sustaineo 2013) que proporciona la participación en certificaciones orgánicas para mejorar las condiciones de vida de los pequeños productores de café de los países del sur.

Adicionalmente, los resultados de este estudio de caso apoyan la argumentación de Beuchelt y Zeller (2011) respecto de los bajos precios que los productores certificados orgánicamente reciben por su café, que en muchos casos se equipara con el de los canales convencionales. En este estudio los productores 
están recibiendo por parte de AMSA entre dos y tres pesos más por quintal que los productores de café convencional. Una situación similar fue ya observada y denunciada por Chiputwa et al. (2013) y el diario La Jornada (Mariscal 2010).

En resumen, este estudio ilustra que la participación en certificaciones orgánicas de café como una estrategia válida hacia la reducción de la pobreza de los pequeños productores en áreas protegidas no está siempre asegurada. No ha servido para fortalecer las estrategias para enfrenar las principales causas de vulnerabilidad de los productores. Las prácticas orgánicas que se realizan en los cafetales tampoco han servido para evitar la propagación de la roya, ni les ha permitido obtener unos precios más altos y justos por su producto. Como menciona Sustaineo (2013), el éxito de esta estrategia depende más de la calidad de los programas que fomentan la participación de los productores en estas certificaciones, que en las certificaciones en sí mismas.

¿Por qué no ha mejorado sustancialmente la calidad de vida de los cafeticultores certificados orgánicamente? Según la opinión de los autores, el principal problema radica en la posición de los productores en la cadena de valor del café, donde son meros generadores de materia prima. El café orgánico se exporta a los países consumidores para posteriormente ser procesado, y es allí donde se concentra gran parte del beneficio. Menos de 10\% de cada dólar gastado en café por un consumidor va a los productores (Daviron y Ponte 2005). Esta situación dentro de la cadena de valor no es cuestionada, ni cambiada por las certificaciones orgánicas. Por consiguiente, es más un problema político que técnico; las certificaciones en sí mismas no pueden cambiar los acuerdos que imperan en el comercio internacional.

En el caso concreto de este estudio podría interpretarse que no solo no se ha cambiado la situación de los productores en la cadena de valor del café, sino que se ha promovido una situación que beneficia en términos económicos y de lavado de imagen 0 "green washing ${ }^{10}$ » a una empresa en detrimento de los PC. Esta circunstancia ha sido nombrada por otros autores como ecocolonialismo o colonialismo verde (Doherty y Doyle 2006, Klein 2016, Kratzer 2015, Machado 2015).

Naomi Klein (2016) y Sebastian Kratzer (2015) mencionan cómo proyectos supuestamente verdes son secuestrados por intereses políticos y económicos más poderosos. Ponen como ejemplo los casos donde poblaciones indígenas se enfrentan al acaparamiento de tierras promovido desde organizaciones conserva-

10 Término que hace referencia a las prácticas de ciertas compañías que mediante acciones de marketing o relaciones públicas promueven una percepción distorsionada de que sus políticas y productos son amigables para el medioambiente. 
cionistas que han convertido en parques naturales y zonas de ecoturismo los ecosistemas indígenas. Estas poblaciones locales se han visto despojadas de sus derechos de uso de la tierra y ya no tienen control sobre los recursos.

\section{El modelo de negocio de café orgánico de Starbucks y Conservation International. Colonialismo verde o solución}

Es necesario mencionar que a raíz de los resultados de este estudio se indagó sobre el modelo que $\mathrm{Cl}$, junto con Starbucks, estableció en la REBISE.

Según Austin y Reavis (2004), en su estudio sobre la asociación entre Cl y Starbucks (la cual empezó en 1998), el proyecto piloto de C.A.F.E Practices se desarrolló tanto en la REBISE como en la reserva colindante El Triunfo. Debido al éxito del proyecto, según $\mathrm{Cl}$ y Starbucks, decidieron exportarlo a otras áreas cafetaleras de América Latina. Situación que también confirma La Jornada: «Santiago Argüello, representante de $\mathrm{Cl}$ en la zona, afirma que la llegada de su organización a la sierra de Chiapas forma parte de una estrategia que abarca toda Mesoamérica y plantea facilitar el mercado a aquellos productores que implementen mejores prácticas de café de conservación» (Mariscal 2004).

La percepción de éxito por parte de Cl y Starbucks contrasta con la opinión de varias de las cooperativas participantes en este modelo de negocio en El Triunfo. Las cooperativas denuncian desavenencias tanto con $\mathrm{Cl}$ como con Starbucks a causa de la inclusión de AMSA en el modelo de exportación y el control por parte de $\mathrm{Cl}$ sobre el proceso de producción, la comercialización, el monopolio del mercado y del financiamiento, al igual que ocurrió en la REBISE (Renard 2008 y Mariscal 2004). Esta situación se ha repetido en los diferentes programas de certificaciones orgánicas de café establecidos por $\mathrm{Cl}$ en asociación con Starbucks en México. Por lo tanto, esto parece apuntar a que las prioridades del modelo de certificaciones orgánicas son única y exclusivamente la exportación de café orgánico de calidad. En los casos mencionados anteriormente la participación de AMSA ha implicado que los productores tengan que vender su café a precios más bajos de lo que se espera de la participación en certificaciones orgánicas. Tampoco se ha puesto especial interés en capacitar y empoderar a los productores para que, a mediano plazo, puedan participar en el mercado internacional de manera independiente: «La posibilidad de que el proyecto de Chiapas ingresara en una nueva etapa de mayor autosuficiencia e independencia de $\mathrm{Cl}$ despertaba 
considerable preocupación y frustración entre el personal de campo que trabajaba con gran entusiasmo en Chiapas» (Austin y Reavis 2004).

En resumidas cuentas, $\mathrm{Cl}$ y Starbucks introdujeron un modelo basado en la certificación del café como orgánico, donde las cooperativas tendrían un mercado seguro y mejor remunerado. Sin embargo, los productores, debido a la dependencia creada con $\mathrm{Cl}$ para obtener las certificaciones y exportar el café a Starbucks, acabaron integrados en un nuevo modelo de negocio donde vendían individualmente su café orgánico a un intermediario o «coyote verde», el cual sustenta la certificación y exporta el café como orgánico pagándole a los productores por debajo del precio esperado en este tipo de mercados.

Según la ONG, este cambio se hizo para reducir los costos de transacción de Starbucks y que este no saliera del negocio. Aunque el hecho de que esto se repitiera en varias zonas, y de que el modelo se quiera exportar a otras áreas cafetaleras, hace pensar que para $\mathrm{Cl}$ y Starbucks es el tipo buscado de negocio. Si esto se confirma parece más bien una expresión de colonialismo verde que para sacar a los pequeños productores de la pobreza.

Doherty y Doyle (2006) mencionan que algunas ONG se han orientado casi exclusivamente hacia donantes externos (en muchos casos grandes compañías transnacionales), dejando de representar los intereses de la gente local. Esto conduce a que se apliquen acciones y políticas que benefician a empresas trasnacionales, a través de ONG ambientalistas, escudándose en acciones en favor de la preservación de la biodiversidad o de lucha contra el cambio climático. El colonialismo verde o ambientalismo como nueva forma de colonialismo corresponde a un enfoque de desarrollo extractivista que, alegando razones de un supuesto manejo responsable de los recursos naturales, pretende mantener una lógica de explotación y mercantilización, en beneficio de los intereses de las empresas trasnacionales. El colonialismo verde contemporáneo no tiene la necesidad de acaparar grandes extensiones de tierra, únicamente necesita mantener el acceso a los productos y a los conocimientos tradicionales que sustenten el desarrollo económico y asegurar el control en un régimen monopólico (Arregi 2011).

No está de más señalar que el director ejecutivo de Starbucks, al igual que otros empresarios como el director ejecutivo de Cemex, forman parte del consejo de administración de Cl (Austin y Reavis 2004). Esta situación lleva a plantearse la existencia de un conflicto de intereses en la ONG ambientalista, al trabajar en proyectos que buscan la mejora de los modos de vida de los pequeños pro- 
ductores de café y, a su vez, tener una influencia tan clara de compañías que no necesariamente comparten esos mismos intereses.

Consecuentemente, parece acertado recomendar estudios que se centren en la situación de los cafeticultores en las cadenas de valor internacionales del café, las causas de su situación vulnerable dentro de la cadena de valor y los requisitos y condiciones para cambiarlas a su favor.

\section{Propuesta de los autores para mejorar la situación de los productores orgánicos en la cadena de valor}

Aumentar el poder de negociación de los productores para así cambiar la situación captiva en la que se encuentran dentro de la cadena de valor.

Permitir el acceso a crédito con buenas condiciones para que puedan pagar el costo de la certificación; de esta forma podrían procesar y comercializar ellos mismos el café pergamino dándole un valor añadido.

Mejorar la implementación de los proyectos por parte de las organizaciones y el gobierno para empoderar a los productores al mejorar sus habilidades organizativas y su conocimiento sobre los mercados internacionales de café. Los proyectos a largo plazo que promuevan el desarrollo de las capacidades de los productores locales y el acceso a crédito parece ser un requisito esencial para el éxito de esta estrategia (Paschall 2013).

Desarrollar una política nacional que apoye claramente a los pequeños productores frente a la situación de libre mercado que favorece a las grandes empresas y a los latifundistas, como parecía ocurrir en la situación previa al desmantelamiento del Instituto Mexicano del Café (INMECAFE), donde las políticas públicas apoyaban y protegían a los cafeticultores mexicanos frente a las situaciones de desigualdad del libre mercado que imperan en el comercio internacional (PérezAkaki 2013).

\section{Reflexiones finales}

En el caso de estudio la estrategia de certificar orgánicamente a los pequeños productores de café solo ha obtenido resultados positivos desde el punto de vista de la conservación y los apoyos puntuales por parte del gobierno. Sin em- 
bargo, no ha tenido impactos relevantes en la mejora de los modos de vida de los productores participantes, ni ha disminuido su vulnerabilidad. El beneficio de la certificación orgánica parece repercutir a todo el resto de la cadena de valor, excepto a los productores: empresas que lo exportan como orgánico (pagándolo a precio convencional), empresas certificadoras, ONG ambientalistas, autoridades de la reserva que buscan cumplir sus metas de conservación (a corto plazo), grandes empresas en los países consumidores que obtienen café de calidad a un precio relativamente bajo, además de beneficiarse de la buena imagen corporativa por vender un producto supuestamente ético y sostenible. Los autores han sugerido que esta situación podría representar un caso de colonialismo verde.

Las posibles soluciones para reducir la vulnerabilidad de los productores pasan por la diversificación de sus ingresos para no ser tan dependientes de un cultivo con precios tan volátiles, tener una cooperativa que realmente funcione con una estrategia de producción y venta colectiva (la falta de capacidad organizativa, confianza entre ellos y una línea de crédito no abusiva no les permite alcanzar dichos objetivos). Por último, la operación de políticas nacionales fuertes que apuesten por los derechos de los pequeños productores cafetaleros frente a las grandes multinacionales.

\section{Reconocimientos}

Expresamos un sincero agradecimiento a los productores de café entrevistados en La Sepultura por participar y compartir sus conocimientos y experiencias. Y a los padres de Iván Soleto, Ignacio y Alma, que hicieron todo posible.

\section{Fuentes de consulta}

Allen, Patricia y Martín Kovach, 2000, "The Capitalist Composition of Organic: The Potential of Markets in Fulfilling the Promise of Organic Agriculture», Agriculture and Human Values, 17, pp. 221-232.

Arregi Joseba, 2011, «Indígenas contra la biopiratería. Una lucha más allá del reconocimiento instrumental de los pueblos indígenas», Revista Observatorio Latinoamericano del Desarrollo Local y la Economía Social, 10(5). 
Austin, James E. y Cate Reavis, 2004, «Starbucks and Conservation International» ${ }_{2}$ Harvard Business School Case 303-055, Boston, Harvard Business School, disponible en $<$ http://www.academia.edu/17305420/Starbucks_and_Conservation_International> [consulta: 3/08/2016].

Bacon, Christopher, 2005, "Confronting the Coffee Crisis: Can Fair Trade, Organic, and Specialty Coffees Reduce Small-Scale Farmer Vulnerability in Northern Nicaragua?», World Development, 33(3), pp. 497-511.

Bacon, Christopher, 2015, «Food sovereignty, food security and fair trade: the case of an influential Nicaraguan smallholder cooperatives», Third World Quarterly, 36(3), 469-488.

Bartra, Armando, Rosario Cobo, Miguel Meza y Lorena Paz, 2003, Sombra y algo más: Hacia un café sustentable mexicano, México, Instituto Maya.

Bartra Armando, 2006, «Virtudes económicas y sociales y ambientales del café certificado: el caso de la Coordinadora Estatal de Café de Oaxaca», en Beatriz Canabal, Gabriela Contreras, Arturo León (coords.), Estrategias económicas y procesos culturales, México, UAM-Xochimilco, pp. 157-202.

Barrett, Hazel, Angela W. Browne, Phil Harris y K. Cadoret, 2002, "Organic certification and the UK market: Organic imports from developing countries», Food Policy, 27, pp. 301-318.

Beuchelt, Tina y Manfred Zeller, 2011, «Profits and poverty: Certification's troubled link for Nicaragua's organic and Fairtrade coffee producers», Ecological Economics, 70, pp. 1316-1324.

Brunel, Marie-Claude y Luis García-Barrios, 2011, «Acknowledging consensus and dissent among and within stakeholder groups over conservation, production and urbanization in a Mexican Man and the Biosphere Reserve», Research Journal of Biological Sciences, 6 (9), pp. 459-467.

Carney, Diana, 1999, Sustainable livelihood approaches: Progress and possibilities for change, Londres, DFID.

Camacho, D.A., J. L. Arellano-Monterrosas, A. C. García, M. García-Castillo, E. G. MuñozCervantes, L. E. Ruiz-Meza, J. R. Trujillo-Vázquez, A. E. Vázquez Robledo y G. Villanueva-López, 2002, Diagnóstico Regional de la Cuenca del Río El Tablón en la Reserva de la Biosfera de La Sepultura, Chiapas, México, San Cristóbal de Las Casas, Universidad Autónoma Chapingo.

Comisión Económica para América Latina y el Caribe (CEPAL), 2002, Centroamérica: El impacto de la caída de los precios del café, Ciudad de México, CEPAL.

Chiputwa, Brian, Matin Qaim y David Spielman, 2013, Food Standards, Certification, and Poverty among Coffee Farmers in Uganda, GlobalFood Discussion Papers, 27.

Cohen, Louis y Lawrence Manion, 1995, Research Methods in Education, Londres, Routledge.

Consejo Nacional de Evaluación de la Política de Desarrollo Social (CONEVAL), 2012, Pobreza estatal Chiapas, disponible en <http://www.coneval.gob.mx/coordinacion/ entidades/Chiapas/Paginas/principal.aspx> [consulta: 20/07/2015].

Daviron, Benoit y Stefano Ponte, 2005, The coffee paradox: Global markets, commodity trade and the elusive promise of development, Londres, Zed. 
Díaz-Bravo, Laura, Uri Torruco-García, Mildred Martínez-Hernández, Margarita VarelaRuiz, 2013, La entrevista, recurso flexible y dinámico, Investigación en Educación Médica, disponible en <http://www.redalyc.org/articulo.oa?id=349733228009> [consulta: 26/04/2017].

Doherty, Brian y Timothy Doyle, 2006, «Beyond borders: Transnational politics, social movement and modern environmentalisms», Environmental Politics, 15(5), pp. 697-712.

González, Alma Amalia y Ronaldo Nigh, 2005, «Smallholder participation and certification of organic farm products in Mexico», Journal of Rural Studies, 21(4), pp. 449-460.

Hallam, David, 2003, Falling commodity prices and industry responses: Some lessons from the international coffee crisis, Roma, FAO.

Kaplinkski, Rapahel y Mike Morris, 2000, A handbook for Value Chain Research, disponible en <http://www.ids.ac.uk/ids/global/VchNov01.pdf> [consulta: 5/07/2015].

Kilian, Bernard, Connie Jones, Lawrence Pratt y Andres Villalobos, 2006, «Is sustainable agriculture a viable strategy to improve farm income in Central America? A case study on coffee», Journal of Business Research, 59(3), pp. 322-330.

Klein, Naomi, 2016, «Let them drown: The violence of othering in a warming world», London review of book, 38(11), pp. 11-14.

Kratzer, Sebastian, 2015, "Climate change and colonialism in the Green Economy», Alternautas, 2(2), pp. 120-125.

Fitter, Robert y Rapahel Kaplinsky, 2001, «Who gains from product rents as the coffee market becomes more differentiated? A value chain analysis», IDS Bulletin, 32, pp. 69-82.

Machado, Horacio, 2015, «Ecología política de los regímenes extractivistas. De reconfiguraciones imperiales y re-exsistencias decoloniales en nuestra América», Bajo el Volcán, 15(23), pp. 11-51.

Mariscal, Ángeles, 2004, «Trasnacional busca controlar el café orgánico: Escudada en grupo ecologista, Starbucks impone condiciones a la venta del grano en Chiapas», La Jornada, 26 abril del 2004, México, disponible en <http://www.jornada.unam. $m \times / 2004 / 04 / 26 / 052 n 1$ con. php?printver=1\&fly> [consulta: 25/07/2016].

Mariscal, Ángeles, 2010, «Nestlé y AMSA ponen en riesgo a caficultores orgánicos en Chiapas», La Jornada, 8 mayo de 2010, México, disponible en <http://www.jornada.unam.mx/2010/05/08/index.php?section=estados\&article=023n1est $>$ [consulta: 25/07/2016].

Parra, Manuel, 2011, Manual de participación comunitaria para promotores y facilitadores del desarrollo comunitario, México, ECOSUR.

Paschall, Melissa, 2013, "The Role of Third Party Certification in Improving Small Farmer Livelihoods», tesis de maestría inédita, University of St. Gallen, School of Management, Economics, Law, Social Sciences and International Affairs.

Pérez-Akaki, Pablo, 2013, «Las políticas públicas cafetaleras en México», Ensayos sobre la economía cafetalera, 29, pp. 121-144.

Ponte, Stefano, 2002, "'Latte Revolution'? Regulation, markets and consumption in the global coffee chain», World Development, 30(7), pp. 1099-1122.

Randall, Laura, 1996, Reforming Mexico's agrarian reform, Nueva York, M. E. Sharpe. 
Renard, Marie-Christine, 2008, "Café de conservación y comercio justo: el caso de las cooperativas de cafeticultores de la reserva de El Triunfo, Chiapas, México», Ponencia presentada en la mesa: Colloque international sur le commerce équitable, en el 3thFair Trade International Symposium, disponible en <http://docplayer.es/4596916-Cafe-de-conservacion-y-comercio-justo-el-caso-de-las-cooperativas-de-cafeticultores-de-la-reserva-del-triunfo-chiapas-mexico.html> [consulta: 1/08/2016].

Schroth, Götz, Peter Laderach y Jan Dempewolf, 2009, «Towards a climate change adaptation strategy for coffee communities and ecosystems in the Sierra Madre de Chiapas, Mexico», Mitig Adapt. Strateg. Glob. Change, 14, pp. 605-625.

Sustaineo, 2013, «Improving smallholder livelihoods: Effectiveness of certification in coffee, cocoa and cotton», KPMG Sustaineo Report.

Thurston, H. David, 1998, Tropical Plant Diseases, St. Paul, MN, American Phytopathological Society.

UNESCO, 2015, «Biosphere Reserves United Nations Educational, Scientific, and Cultural Organization», UNESCO, disponible en <http://www.unesco.org/new/en/naturalsciences/environment/ecological-sciences/biospherereserves/> [consulta: 19/02/2015].

Wild, Anthony, 2005, Coffee: A dark history, Nueva York, Norton. 